\title{
Study on Patterns of Human Cancer Using SSN Method
}

\author{
Chaoyu Zhang \\ Xi'an Jiaotong University, Xi'an, China \\ Email: 723836984@qq.com
}

How to cite this paper: Zhang, C.Y. (2020) Study on Patterns of Human Cancer Using SSN Method. Open Access Library Journal, 7: e6453.

https://doi.org/10.4236/oalib.1106453

Received: May 23, 2019

Accepted: June 21, 2020

Published: June 24, 2020

Copyright $\odot 2020$ by author(s) and Open Access Library Inc.

This work is licensed under the Creative Commons Attribution International License (CC BY 4.0).

http://creativecommons.org/licenses/by/4.0/

(c) (i) Open Access

\begin{abstract}
Human cancer, which has complex pathogenesis, is generally relative to the dysfunction of biological systems. Thus, our research is not at molecular level, but at system level, i.e. molecular network. In this paper, specially, we use PPI network. In order to construct a PPI network, we used the SSN method which is proposed by Professor X. Liu and others. The SSN method is distinct from the traditional network methods, especially in screening differential expressed genes. Besides, the traditional network method cannot show characters of disease of every sample. However, the SSN method has this ability because of its unique character. The main purpose of this thesis is to analyze the high-throughput sequencing data of 8 human cancers by using the SSN method. We programmed to realize most of the research and the other part was realized by web tools. Our work included introducing the SSN method theoretically and analyzing data of human cancer by the SSN method. It was proved that the SSN method was feasible and reliable in the study of human cancer in this thesis. The SSN method proposes a new way in construction of biomolecular networks, which is a great promotion.
\end{abstract}

\section{Subject Areas}

Bioinformatics, Mathematical Statistics

\section{Keywords}

Cancer, Molecular Network, SSN Method, Traditional Network Method

\section{1. 引言}

人类癌症的产生原因往往和基因的变化密不可分, 而单个或是几个基因的变 化不一定会导致癌症的产生。癌症的产生机制十分复杂[1] [2], 与生物系统层 面的变化相关。本文目的就是从系统层面研究与癌症相关的关键基因。因此, 
我们构建了分子网络也就是文中的蛋白质互作网络来反应系统层面癌症的特 性。SSN 方法[3]是本文用来构建蛋白质互作网络的方法, 它有着传统网络方 法不具备的优点。传统网络方法主要是利用基因测序结点的表达数据来笁选 差异基因、构建网络的, 这样做也确实能反应出一些和癌症有关的关键基因, 但是由于此方法只将样本分为总的两类进行比对, 即正常样本和癌症样本, 因此无法关注每一个癌症样本的表达模式。然而, SSN 方法使用正常样本作 为对照, 可以构建每个癌症样本的蛋白质互作网络。这么做的好处是我们可 以具体关注每个癌症样本的癌症表达模式, 以此实现针对每个癌症个体的个 性化医疗。

高通量测序技术, 也称下一代测序技术[4], 有着测序准确度高、测序效率 高以及测序成本低等优势。测序技术的发展, 使得获取大量的基因表达数据 成为了现实。本文主要的研究数据就是基因表达数据, 我们从 TCGA 数据库 [5]下载了 8 种人类癌症的高通量测序[4]数据用以 SSN 的构建。在构建 SSN 时, 我们先用 Matlab 进行数据分析和预处理, 之后使用了 String 网页工具来 获得蛋白质互作网络图[6]。构建出 SSN 后, 我们使用 Cytoscape 软件[7]提取 各癌症样本的 TP53 子网。TP53 子网是我们重点关注的对象之一, 这是由于 TP53 基因是为制造 p53 这种蛋白质提供指令的基因, 而 p53 蛋白质的作用就 是抑制肿瘤, 也就是说 TP53 基因与癌症的产生是息息相关的 [8] [9]。后续我 们对比了 SSN 方法和传统网络方法在分类正常样本和癌症样本时的情况, 其 中 SSN 方法通过 Matlab 编程实现差异基因的篮选, 而传统网络方法通过 NetworkAnalyst 网页工具[10]实现差异基因的篮选。䇥选完差异基因后我们提 取了前五位的差异基因, 通过 Heatmapper 网页工具[11]进行热图的制作以及 分类准确率的统计。

我们使用 SSN 方法完成对癌症表达模式的分析, 是对传统网络方法的一种 改进与提升, 并且我们也验证了 SSN 方法的可用性和可靠性。

\section{SSN}

\subsection{SSN 简介}

对于人类而言, 大多数疾病的产生机制、产生原因都是复杂的, 尤其是对于 发病原因仍未明了的癌症来说。这些复杂的疾病不单单由一个分子或者几个 分子的功能失常引起, 而是由于系统的失常或者分子网络的失常导致的。因 此, 如果想要对疾病的产生机制进行研究, 我们主要的研究对象应当是系统 或者分子网络。因此, 本文的主要研究对象就是分子网络, 我们利用了分子 网络来反应系统层面的癌症的表达模式。分子网络是一种分子之间相互作用 的关系网络, 它用来反应分子之间相互连接的关系。分子网络包括了生物代 谢与信号传导网络、基因转录调控网络以及蛋白质互作网络, 其中本文使用 的分子网络是蛋白质互作网络。蛋白质互作网络是由单独的蛋白通过彼此之 间的相互联系、表达作用形成的网络，它能反映不同基因表达的蛋白质之间 联系的强弱, 我们可以用它来反映系统层面疾病的性质。图 1 是大肠杆菌有 机体系中四种蛋白质相互联系形成蛋白质互作网络的例子。每个结点 $\operatorname{trpA}$ 、 $\operatorname{trpB} 、 \operatorname{trpC} 、 \operatorname{trpD}$ 均表示一种蛋白质, 结点之间的连线(边)代表蛋白质之间的 联系关系。此外, 连接两个结点的边还能用来反应这两种蛋白质联系的强弱 性质, 边越粗说明它们之间的联系越强。 


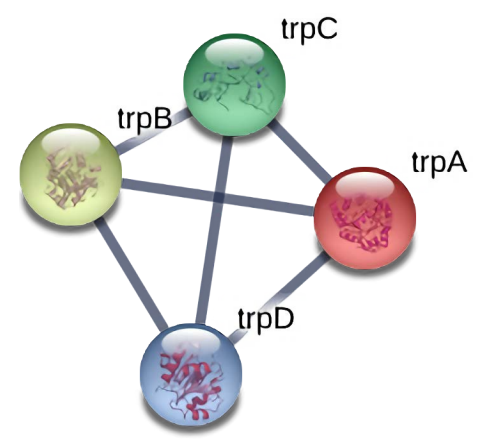

图 1. 蛋白质互作网络示例。

为了构造癌症的蛋白质互作网络, 我们需要大量的基因表达信息, 这完全 得益于高通量测序技术。高通量测序技术, 即第二代测序技术, 是一种对基 因测序、获取基因表达信息的测序方法。第二代测序技术对比第一代测序技 术, 有着可靠性更高、测序成本低廉、一次能测定几百万条 DNA 分子等优点。 也正是由于测序技术近些年的飞速发展, 人们越来越热衷于研究分子网络, 尤其是对于疾病的分子网络的研究。并且用分子网络来阐明疾病系统层面的 一些特质也正受着越来越多的关注。因此, 本文的最终目的是利用不同癌症 的基因测序信息构造癌症样本的分子网络, 然后通过研究这些分子网络, 来 寻找不同种类的癌症是否具有不同的、独特的网络模式, 并且尝试通过分子 网络去寻找癌症发病的一些关键因素。

SSN 就是本文用来研究癌症疾病模式所构造的蛋白质互作网络。SSN 是基 于一组正常样本对照所构建出来的蛋白质互作网络。高通量测序数据中, 存 在癌症样本和正常样本, 一个正常样本的数据代表从一个正常人身上提取 mRNA 进行测序获得的数据, 一个癌症样本的数据代表从一个患癌的人身上 提取 mRNA 进行测序获得的数据, 样本的数量代表的就是人的数量。为了构 建 SSN, 我们先利用一定数量的正常人的样本作为对照, 通过基因之间的相 关性, 我们以这些作为对照的正常样本为基础构建一个网络, 我们将这个网 络命名为参考网络。这个参考网络只是构建 SSN 中用来过渡的网络, 因此不 需要将它具体化 (用结点和边的形式画出来)。为了获得正常样本中两个基因之 间的相关性, 我们通过计算两个基因之间的皮尔逊相关系数, 以此来量化相 关性。只要得到所有基因的两两对应的相关性, 那么显然我们就能构建这些 基因表达的蛋白质所对应的蛋白质互作网络, 即参考网络。接着我们引入了 一个癌症样本, 再计算正常样本加入癌症样本后的两个基因的相关系数, 如 果相关系数变化很大, 那么我们可以初步认为这两基因的变化与这个样本癌 症的产生有关, 这是因为正常样本有共同的基因表达模式, 即使有差别相关 性也不会变化很多。我们将以正常样本加上一个癌症样本为基础构成的网络 称为扰动网络。同样这个扰动网络也只是构建 SSN 中用来过渡的网络, 因此 也不需要将它具体化(用结点和边的形式画出来)。如上过程重复对每个癌症样 本进行处理, 如果大部分的癌症样本里的这两个基因都有这种相关性的变化, 那么不难推断这两个基因很有可能是导致正常样本变成癌症样本的基因。通 过篮选这一部分基因, 我们就能获得与癌症产生相关的关键基因。

这个方法里, 正常样本作为对照使用, 实际上我们构建网络使用的样本就 仅仅只有一个癌症样本。与传统构建网络的方法对比之下, 传统构建网络的 
方法往往需要大量的样本。并且在篮选差异基因部分, 传统网络方法使用的 是对结点进行篮选, SSN 方法使用的是对边进行筛选, 据资料显示, SSN 方 法能篮选出一些传统网络方法不能笁选出的关键基因, 这也是 SSN 方法相对 于传统网络方法的优点。传统网络方法是将正常样本和癌症样本全部分类放 在一个矩阵, 比如 1 到 20 列是正常样本的表达数据, 21 到 40 列是癌症样本 的表达数据。每一行代表一个基因测序结点, 通过逐行计算正常样本和癌症 样本之间值的倍数 FoldChance 简记为 FC, 和检验 $p$ 值以及通过一些方法修 正的 $p$ 值, 然后通过框定条件:

$$
\begin{gathered}
\left|\log _{2} F C\right|>1 \\
p<0.05
\end{gathered}
$$

筛选出关键的差异基因, 以此来构建蛋白互作网络。相对于传统网络方法, SSN 方法最大的特点还是在笁选差异基因的时候只使用了一个癌症样本。由 于 SSN 方法仅仅使用一个癌症样本来研究癌症的疾病模式, 并且对于每种癌 症的每个癌症样本均能生成一个对应的蛋白质互作网络, 所以我们将这个用 扰动网络减去参考网络得到的差异网络命名为单样本网络(Single Sample Network), 这也是 SSN 这个名字的由来。这里差异网络的意思就是扰动网络 减去参考网络得到网络的名称, 因为由两个网络表达量之差得来, 所以称之 为差异网络。如果我们对每个癌症样本都去计算基因之间的两两相关性, 意 味着实际上我们可以对每个癌症样本构建差异网络。这是 SSN 方法相对于传 统网络方法的优势之处, 这也是我们使用 SSN 方法的很重要的原因。由 SSN 方法我们可以得到每个样本自己独特的、特异性的表达模式, 因此我们也将 这个差异网络称为样本特异性网络(Sample Specific Network)。

综上所述, 我们使用 SSN 方法的目的, 就是建立一个通过一系列数据处理 得到的分子网络来对人类癌症疾病模式进行研究, 以此实现对传统网络方法 的一种补充、改进, 弥补传统网络方法在样本需求高、笁选差异基因构建蛋 白互作网络等方面的一些缺陷。

\section{2. 背景知识}

\subsection{1. 皮尔逊相关系数 PCCS}

构建 SSN 时, 为了将两个基因之间的相关性量化, 我们引入皮尔逊相关系 数来计算, 通过数值定量反应基因之间的相关性。

皮尔逊相关系数是用来计算两个变量之间线性相关性的一个值, 它的定义 是两个变量之间的协方差与标准差的商。

$$
P C C=\frac{\operatorname{cov}(X, Y)}{\sigma_{x} \sigma_{y}}=\frac{E\left[\left(X-\mu_{x}\right)\left(Y-\mu_{y}\right)\right]}{\sigma_{x} \sigma_{y}}
$$

假设有两个变量 $X 、 Y$, 如果 $X 、 Y$ 正相关, 那么它们的皮尔逊相关系数等 于 1; 如果 $X 、 Y$ 负相关, 那么它们的皮尔逊相关系数等于 -1 ; 如果皮尔逊相 关系数等于 0 , 那么 $X 、 Y$ 不相关。本文主要使用皮尔逊相关系数的绝对值, 如果皮尔逊相关系数的绝对值向 0 靠拢, 那么两个变量的相关性减小; 如果 皮尔逊相关系数的绝对值向 1 靠拢, 那么两个变量的相关性增加。

本文中参考网络由 $n$ 个正常样本构成, 那么我们定义基于这 $n$ 个样本的两 个基因的相关系数为 $P C C_{n}$, 于是在参考网络中连接这两个基因的边对应的 
值就为 $P C C_{n}$; 而扰动网络是由 $n$ 个正常样本以及 1 个癌症样本构成, 那么 我们定义基于这 $n+1$ 个样本的两个基因的相关系数为 $P C C_{n+1}$, 于是在参考 网络中连接这两个基因的边对应的值就为 $P C C_{n+1}$ 。差异网络是扰动网络减去 参考网络而来的网络, 因此在差异网络中连接这两个基因的边的对应值就为 $P C C_{n+1}-P C C_{n}$, 记为 $\triangle P C C_{n}$ 。

\subsection{2. $t$ 检验}

在说明 $P C C_{n}$ 分布情况时, 可以用自由度为 $n-2$ 的检验来计算 $P C C_{n}$ 的 $p$ 值, 检验统计量为

$$
t=\frac{P C C_{n}}{\sqrt{\frac{1-P C C_{n}^{2}}{n-2}}}
$$

其中 $p$ 值的意思是落在拒绝域的概率。本文作出了当 $n=100$ 时 $P C C_{n}$ 的分布 情况, 即概率密度函数, 如图 2 所示。当 $n$ 增加的时候, $P C C_{n}$ 的分布会越 来越趋近正态分布, 于是我们猜测, 当 $n$ 趋向于无穷的时候, $P C C_{n}$ 的分布 无限接近于正态分布。

\subsection{3. $u$ 检验}

本文中使用 $u$ 检验来检验 SSN 中的边是否显著，如果检验 $p$ 值小于 0.05 , 那 么意味着落在拒绝域的概率很小, 则认为这条边在 SSN 中显著; 反之如果检 验 $p$ 值大于 0.05 , 则认为这条边在 SSN 中不显著。一条边在 SSN 中显著也 就意味着这条边在 SSN 中是存在的。本文中一些地方, 比如绘制人类 8 种不 同癌症的各 10 个随机癌症样本的 TP53 子网, 篮选差异基因时, 由于篮选出 的差异基因数量超出了 String 网页工具能处理的数量, 我们将 $P$ 值范围区分 为小于 0.01 和大于 0.01 , 小于 0.01 则边在 SSN 中显著, 大于 0.01 则边在 SSN 中不显著。

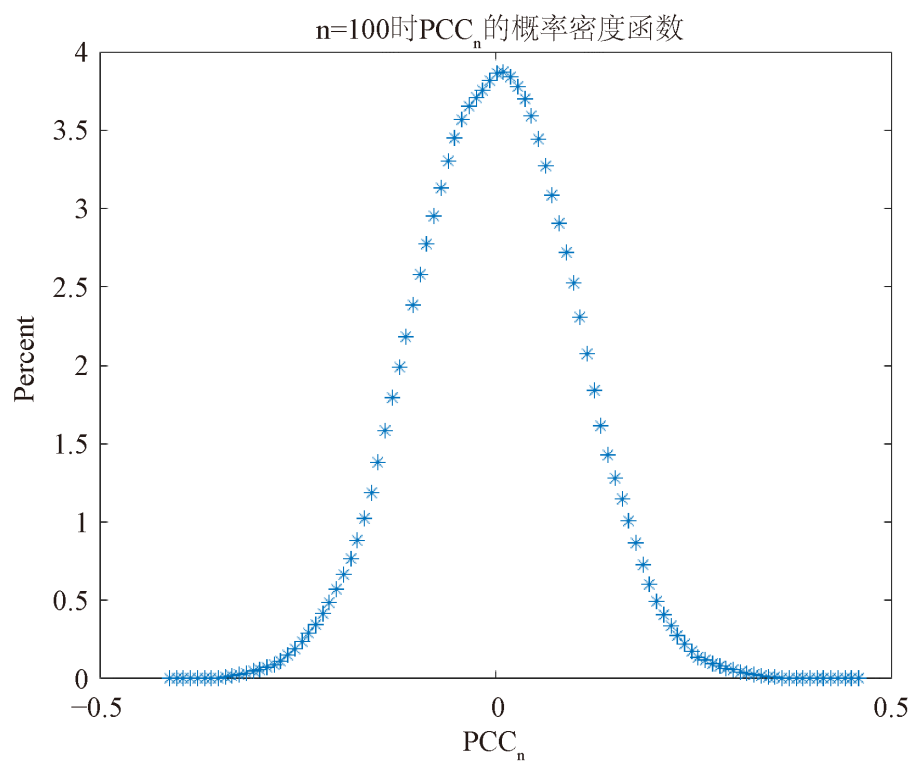

图 2. $n=100$ 时 $P C C_{n}$ 的概率密度函数。 
因为 $P C C_{n}$ 的分布在 $n$ 比较大的时候趋向于正态分布, 那么显然 $P C C_{n+1}$ 也 是趋向于正态分布的。

$$
\triangle P C C_{n}=P C C_{n+1}-P C C_{n}
$$

我们通过检验 $\triangle P C C_{n}$ 与 $\triangle P C C_{n}$ 的总体均值 0 是否相等来判断一条边是否显 著, 实际上我们判断的是 $P C C_{n}$ 到 $P C C_{n+1}$ 的变化是否显著。因此我们实际上 进行的是两个正态总体值的假设检验, 即检验的 $H_{0}$ 为 $u_{1}=u_{2}, H_{1}$ 为 $u_{1} \neq u_{2}$. 那么我们可以将其转变为 $H_{0}$ 是 $u_{1}-u_{2}=0, H_{1}$ 是 $u_{1}-u_{2} \neq 0$, 于是这就变成了 单个正态总体的假设检验, $u_{1}-u_{2}$ 就是 $P C C_{n+1}-P C C_{n}$, 于是假设检验就变为 验 $\triangle P C C_{n}$ 是否等于 0 。

单个正态总体的假设检验中, $u$ 检验的检验统计量为

$$
u=\frac{\bar{X}-\mu}{\sigma / \sqrt{n}}
$$

本文使用的 $u$ 检验为双边 $u$ 检验，如图 3 所示，正态分布的双尾区域(打阴 影部分) 即为拒绝域, 落在这两部分的概率即为 $p$ 值。我们知道正态分布的概 率密度函数总面积为 1 , 即整个图的面积为 1 , 那么 $p$ 值就等于落在双尾区域 阴影部分的面积的值。

\section{3. 构建单个癌症样本的 SSN}

SSN, 代表单样本网络(Single Sample Network), 也代表样本特异性网络 (Sample Specific Network)。由于癌症产生的原因是复杂的、系统性的, 我们 需要构建不同种类癌症的分子网络, 观察不同的癌症是否有不同的网络模式, 并且通过构建的分子网络来寻找与发病有关的的基因或是蛋白质。SSN 就是 本文我们构建的用来研究癌症疾病模式的分子网络, 也就是蛋白质互作网络。

为了构建一个癌症样本的 SSN, 我们首先需要构建一个基于普通人的用以

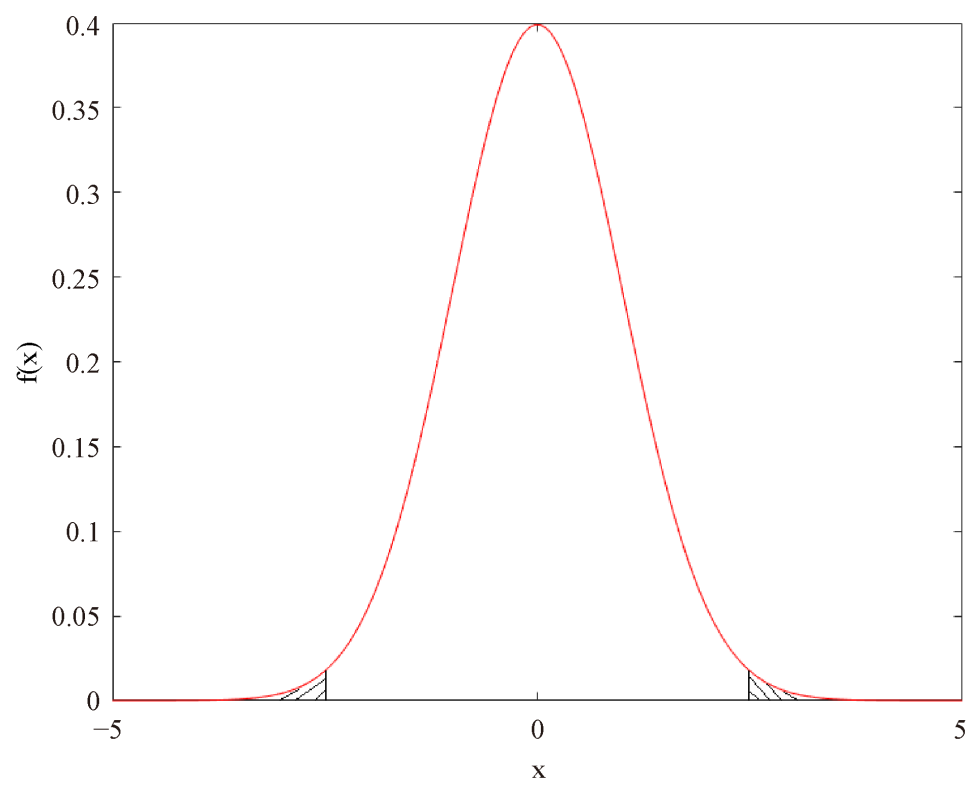

图 3. 正态分布的概率密度函数。 
对照的网络, 即参考网络。参考网络是由一组正常的样本为基础构建得来的, 正常样本的选取数量在 10 20 个左右, 由于一些癌症的正常样本数不多, 于 是选取的正常样本数量极个别会有例外。参考网络的正常样本数如果太多, 那么计算的速度就会减慢; 数量如果太少, 那么在计算基因之间的相关系数 以及判断相关系数是否显著变化的时候, 精确度就会降低。由于参考网络中 所有的样本都是正常样本, 因此参考网络是具有正常样本公共属性的网络。 我们构建参考网络的目的是将参考网络作为扰动网络的对照。扰动网络指的 是通过在正常样本种加入一个癌症样本, 以这些样本为基础构建的网络。我 们将扰动网络去和参考网络作比较, 观察在两个网络中连接两个相同基因的 边是否有显著的变化。如果有显著的变化, 那么我们初步认为这个变化和癌 症的产生是有关的; 如果没有显著的变化, 就认为与癌症的产生无关。对照 的网络需要是正常的、普遍的, 因此构成参考网络的样本中不可以有癌症样 本。

我们从 TCGA 数据库下载下来 TCGA 数据后, 经过 Excel 简单处理的结果 一般如图 4 中样本 123 构成的表所示所示, 第一行代表的是样本的名称, 这 里用 s1、s2 …来表示, 第一列代表的是基因测序节点, 这里用 g1、g2 …… 来表示。因为样本中正常和癌症样本是穿插出现的, 所以我们要先对正常样 本以及癌症样本分类, 分类详细过程见数据预处理部分。分类完正常样本与 癌症样本后, 我们就可以选择一定数量的正常样本通过皮尔逊相关系数来构 建参考网络。当一个癌症样本 (图 4 中的 $\mathrm{d}$ 样本) 被加入参考网络的时候, 我 们称这个加入后由皮尔逊相关系数构成的网络为扰动网络。由此, 我们可以 得到参考网络以及扰动网络之间的差异网络, 并且参考网络与扰动网络之间 的差异仅仅在于癌症样本 $\mathrm{d}$ 。

统计学中, 皮尔逊相关系数 PCC 被用来度量两个变量 $X 、 Y$ 之间的相关性, 参考网络中两个基因(比如 g1 与 g2)之间的相关性就由皮尔逊相关系数决定， 记为 $P C C_{n}$ ( $n$ 个样本的皮尔逊相关系数)。同理扰动网络两个基因之间的相关 性就由皮尔逊相关系数决定, 记为 $P C C_{n+1}(n+1$ 个样本的皮尔逊相关系数)。 如果加入的样本 $\mathrm{d}$ 在基因表达的形式上与正常样本差距不大, 那么 $P C C_{n}$ 和 $P C C_{n+1}$ 之间不会有显著的差异; 如果加入的样本 $\mathrm{d}$ 在表达形式上与正常样本 差距显著, 那么 $P C C_{n}$ 和 $P C C_{n+1}$ 之间会有显著的差异。因此我们主要关注的 是 $P C C_{n}$ 和 $P C C_{n+1}$ 之间的差异, 即 $\triangle P C C_{n}\left(\triangle P C C_{n}=P C C_{n+1}-P C C_{n}\right)$, 以此 来构建一个差异网络。这个差异网络就是仅使用了一个癌症样本 $\mathrm{d}$ 构建出来 的 SSN, 我们称它为样本 $\mathrm{d}$ 的 SSN。此外, 这个 SSN 只由样本 $\mathrm{d}$ 构成, 所以 它能单独反应样本 $\mathrm{d}$ 的癌症的疾病状况, 这一点是传统网络方法不具备的性 质。构建单个癌症样本 SSN 的算法流程图如图 5 所示。

\section{4. 理论基础}

\subsubsection{PCC 的分布情况}

要说明 SSN 的理论基础, 我们首先要了解 PCC 的分布情况。我们假设有 $n$ 个样本, 两组基因表达 $X$ 与 $Y$ 。两组基因表达分别为 $X=\left(x_{1}, x_{2}, \cdots, x_{n}\right)$, $Y=\left(y_{1}, y_{2}, \cdots, y_{n}\right)$, 其中 $x_{i}(1 \leq i \leq n)$ 代表基因 $X$ 在第 $i$ 个样本中的表达量, $y_{i}(1 \leq i \leq n)$ 代表基因 $Y$ 在第 $i$ 个样本中的表达量。根据皮尔逊相关系数的定 义, 这里 

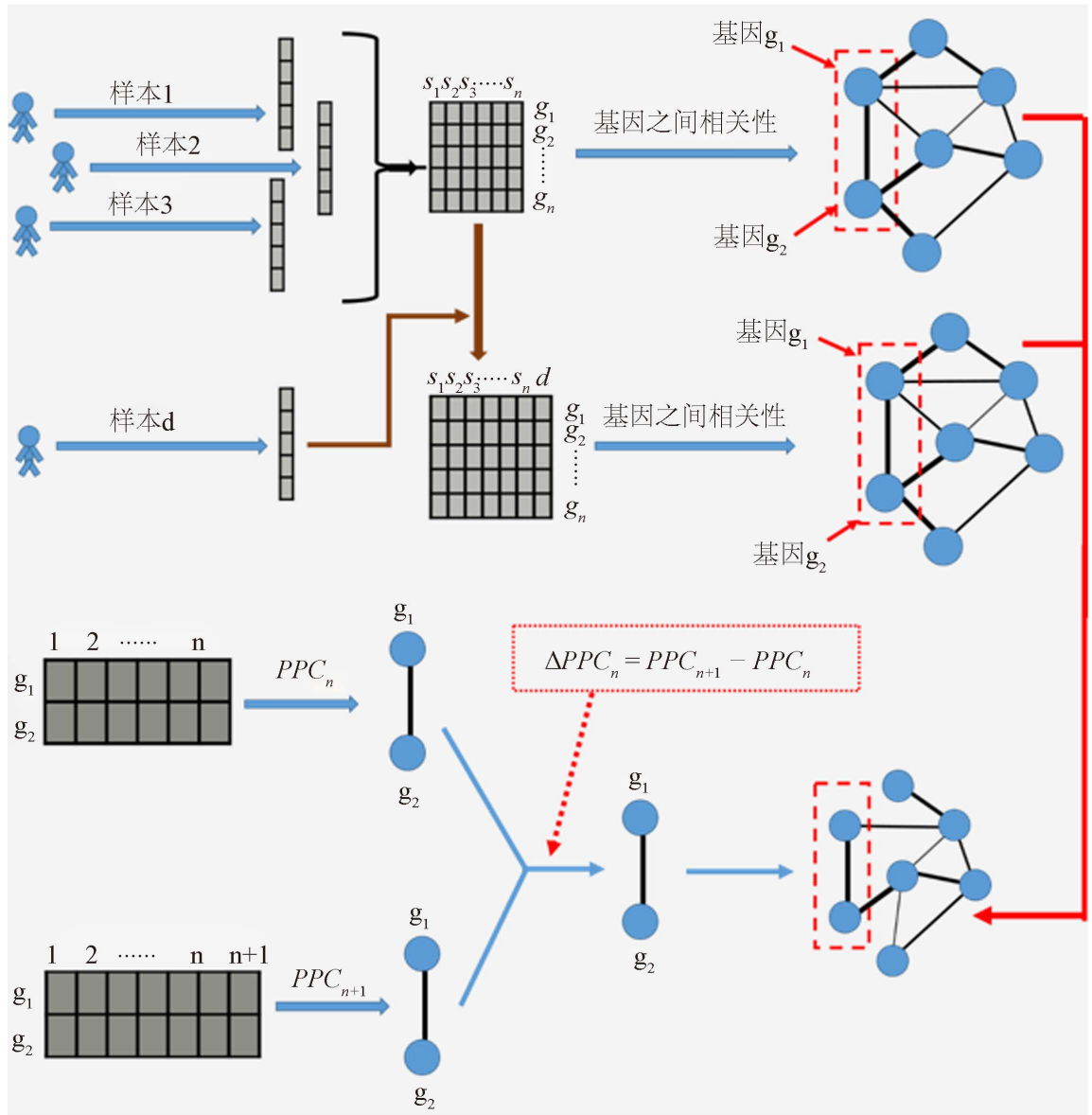

图 4. 构建一个癌症样本 d 的 SSN。

$$
\operatorname{PCC}_{n}=\frac{\sum_{i}^{n}\left(x_{i}-\overline{x_{n}}\right)\left(y_{i}-\overline{y_{n}}\right)}{\sqrt{\sum_{i=1}^{n}\left(x_{i}-\overline{x_{n}}\right)^{2}} \sqrt{\sum_{i=1}^{n}\left(y_{i}-\overline{y_{n}}\right)^{2}}}
$$

其中

$$
\begin{aligned}
& \overline{x_{n}}=\frac{1}{n} \sum_{i=1}^{n} x_{i} \\
& \overline{y_{n}}=\frac{1}{n} \sum_{i=1}^{n} y_{i}
\end{aligned}
$$

那么可以得到 $P C C_{n}$ 的期望为 0 , 方差为 $1-P C C_{n}^{2}$ 。因此, $P C C_{n}$ 的 $p$ 值可 以通过自由度为 $n-2$ 的 $t$ 检验来计算得到, 假设检验统计量为

$$
t=\frac{P C C_{n}}{\sqrt{\frac{1-P C C_{n}^{2}}{n-2}}}
$$

2.4.2. $\triangle P C C_{n}$ 的分布情况

由于 SSN 是差异网络, 主要研究的是 $\triangle P C C_{n}$ 的情况, 所以我们要进一步说明 


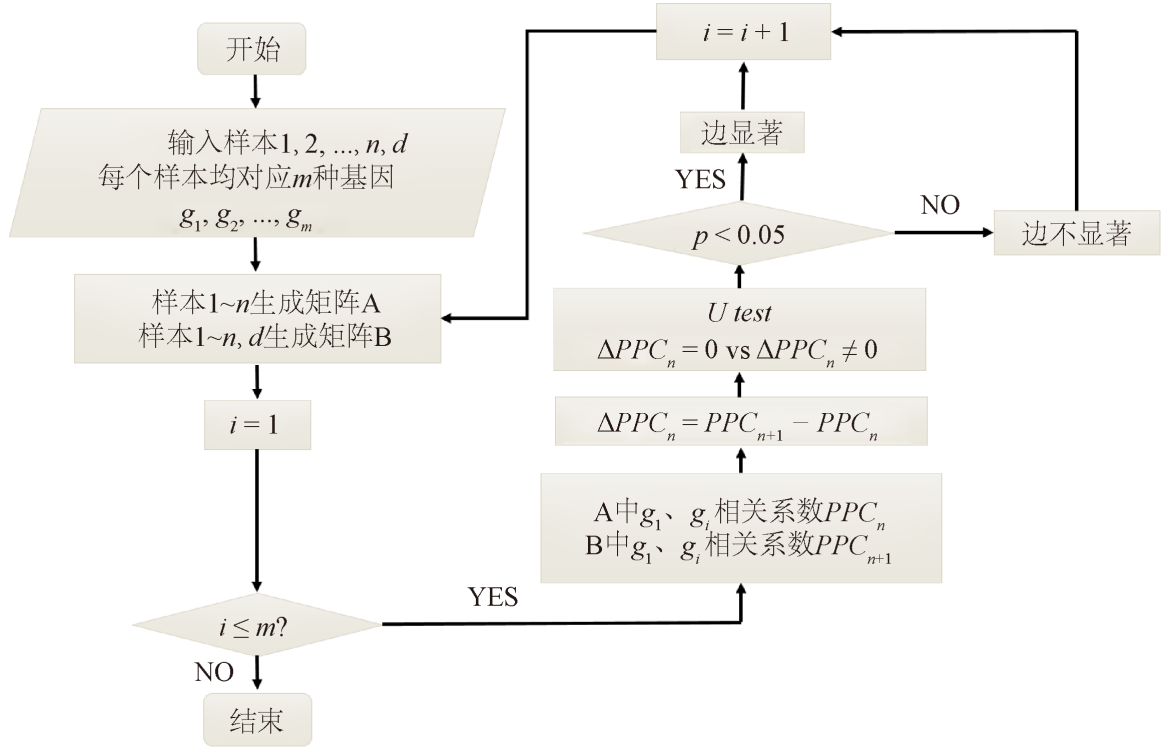

图 5. 构建癌症样本 d 的 SSN 的流程图。

$\triangle P C C_{n}$ 的分布情况。我们假设有 $n$ 个样本, 两组基因表达 $X$ 与 $Y$ 。两组基因 表达分别为 $X=\left(x_{1}, x_{2}, \cdots, x_{n}\right), Y=\left(y_{1}, y_{2}, \cdots, y_{n}\right)$, 其中 $x_{i}(1 \leq i \leq n)$ 代表基因 $X$ 在第 $i$ 个样本中的表达量, $y_{i}(1 \leq i \leq n)$ 代表基因 $Y$ 在第 $i$ 个样本中的表达 量。根据皮尔逊相关系数的定义, 这里

$$
\operatorname{PCC}_{n}=\frac{\sum_{i}^{n}\left(x_{i}-\overline{x_{n}}\right)\left(y_{i}-\overline{y_{n}}\right)}{\sqrt{\sum_{i=1}^{n}\left(x_{i}-\overline{x_{n}}\right)^{2}} \sqrt{\sum_{i=1}^{n}\left(y_{i}-\overline{y_{n}}\right)^{2}}}
$$

其中

$$
\begin{aligned}
& \overline{x_{n}}=\frac{1}{n} \sum_{i=1}^{n} x_{i} \\
& \overline{y_{n}}=\frac{1}{n} \sum_{i=1}^{n} y_{i}
\end{aligned}
$$

当加入一个样本 $\mathrm{d}$ 后, 我们假设 $X=\left(x_{1}, x_{2}, \cdots, x_{n}, x_{n+1}\right)$, $Y=\left(y_{1}, y_{2}, \cdots, y_{n}, y_{n+1}\right)$, 其中 $x_{i}(1 \leq i \leq n+1)$ 代表基因 $X$ 在第 $i$ 个样本中的表 达量, $y_{i}(1 \leq i \leq n+1)$ 代表基因 $Y$ 在第 $i$ 个样本中的表达量。根据皮尔逊相关 系数的定义, 这里

$$
\operatorname{PCC}_{n+1}=\frac{\sum_{i}^{n}\left(x_{i}-\overline{x_{n+1}}\right)\left(y_{i}-\overline{y_{n+1}}\right)+\left(x_{n+1}-\overline{x_{n+1}}\right)\left(y_{n+1}-\overline{y_{n+1}}\right)}{\sqrt{\sum_{i=1}^{n}\left(x_{i}-\overline{x_{n+1}}\right)^{2}+\left(x_{n+1}-\overline{x_{n+1}}\right)^{2}} \sqrt{\sum_{i=1}^{n}\left(y_{i}-\overline{y_{n+1}}\right)^{2}+\left(y_{n+1}-\overline{y_{n+1}}\right)^{2}}}
$$

其中

$$
\overline{x_{n+1}}=\frac{1}{n+1} \sum_{i=1}^{n+1} x_{i}
$$




$$
\overline{y_{n+1}}=\frac{1}{n+1} \sum_{i=1}^{n+1} y_{i}
$$

通过线性化等一系列处理可以得到 $\triangle P C C_{n}$ 的期望为 0 , 标准差为 $\frac{1-P C C_{n}^{2}}{n-1}$ 。

因此, 根据中心极限定理, 即统计学中用来分析随机变量序列的分布服从 正态分布的定理, 我们可以使用 $u$ 检验(即 $z$ 检验) 来估计 $\triangle P C C_{n}$ 的显著性。 通过程序展现、比较 $\triangle P C C_{n}$ 的分布与正态分布, 我们可以看出分布的双尾区 域是相似的。图 6(a)展示了 $n=100$ 时 $\triangle P C C_{n}$ 的分布(红线)和正态分布(蓝线) 的概率密度函数, 当 $n$ 增加时, $\triangle P C C_{n}$ 的分布与正态分布越来越接近, 如图 6(b)。于是我们认为当 $n$ 趋向于无穷的时候, 对于双尾区域来说, $\triangle P C C_{n}$ 的 分布无限接近于正态分布。我们将图中红线的分布命名为 “火山分布”。由 于在做假设检验的时候, 我们用的是双边 $u$ 检验, 因此当双尾区域相似时, 我们可以简单地将这种 “火山分布” 当作是正态分布分析, 这是我们重点关 注双尾区域的原因。

因为我们要研究每条边的显著性, 所以我们对每个 $\triangle P C C_{n}$ 都要进行假设检 验。原假设为 $\triangle P C C_{n}$ 等于 $\triangle P C C_{n}$ 的总体均值, 这里 $\triangle P C C_{n}$ 的总体均值取 0 。 假设检验统计量为

$$
u=\frac{\Delta P C C_{n}}{\left(1-P C C_{n}^{2}\right) /(n-1)}=\frac{(n-1) \Delta P C C_{n}}{1-P C C_{n}^{2}}
$$

如果假设检验得到的 $p$ 值小于 0.05 , 那么我们认为 $\triangle P C C_{n}$ 所对应的这条边是 显著的, 即这条边在我们构建的 SSN 中是存在的; 否则我们认为这条边不显 著且不存在于我们构建的 SSN 中。

为了验证我们得到的 $u$ 检验统计量, 我们随机生成两组数当作表达数据 $X=\left(x_{1}, x_{2}, \cdots, x_{n}\right), Y=\left(y_{1}, y_{2}, \cdots, y_{n}\right)$ 。长度 $n$ 由 5 变化到 $200, X 、 Y$ 之间的 皮尔逊相关系数 PCC 从 0 变化到 0.9 。对于每一对 $n$ 和 PCC, 都随机模拟 20000 次, 每次模拟都选取 “火山分布” 双尾区域中 $p$ 值等于 0.05 时的 $\triangle P C C_{n}$ 的值, 并且根据正态分布理论计算, 我们也能得到 $\triangle P C C_{n}$ 相对应的理论值。通过比 较随机模拟的 $\triangle P C C_{n}$ 的值和理论 $\triangle P C C_{n}$ 的值, 如图 6(c), 我们发现在 $n$ 比较

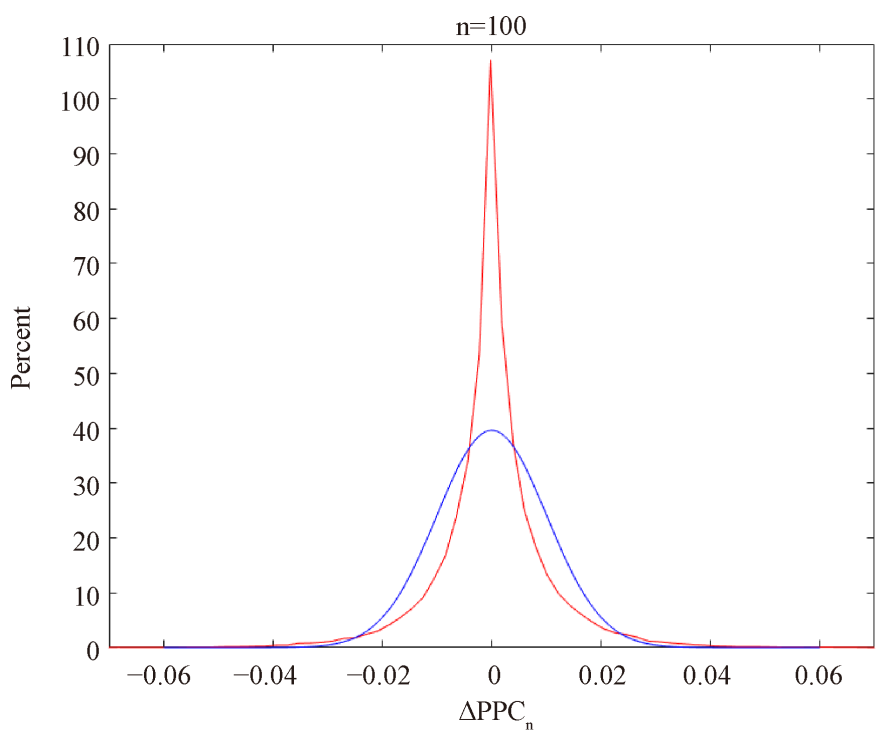

(a) $n=100$ 时的分布情况 


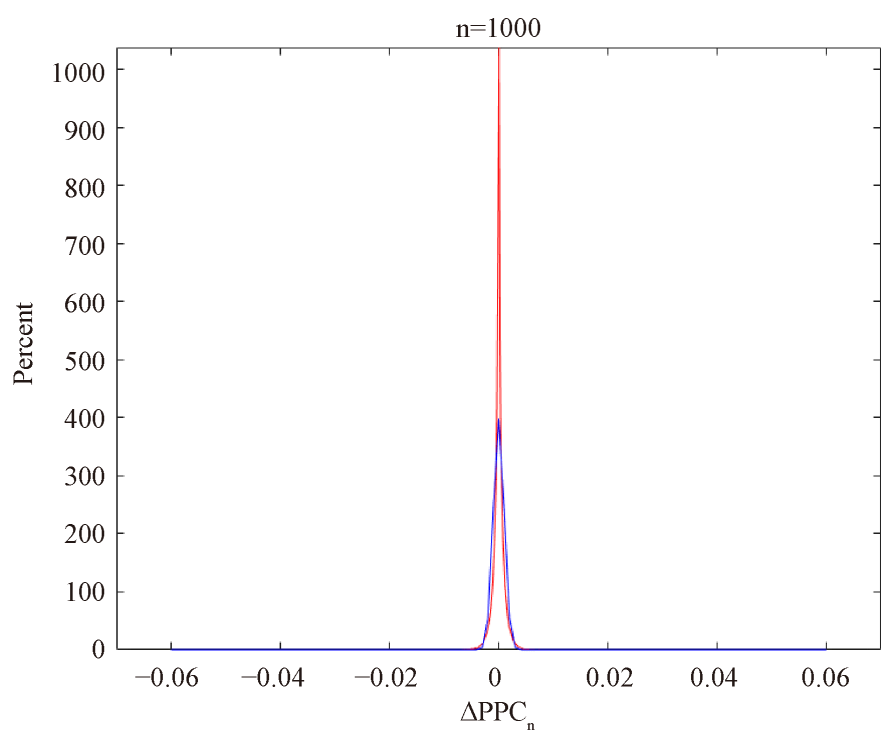

(b) $n=1000$ 时的分布情况

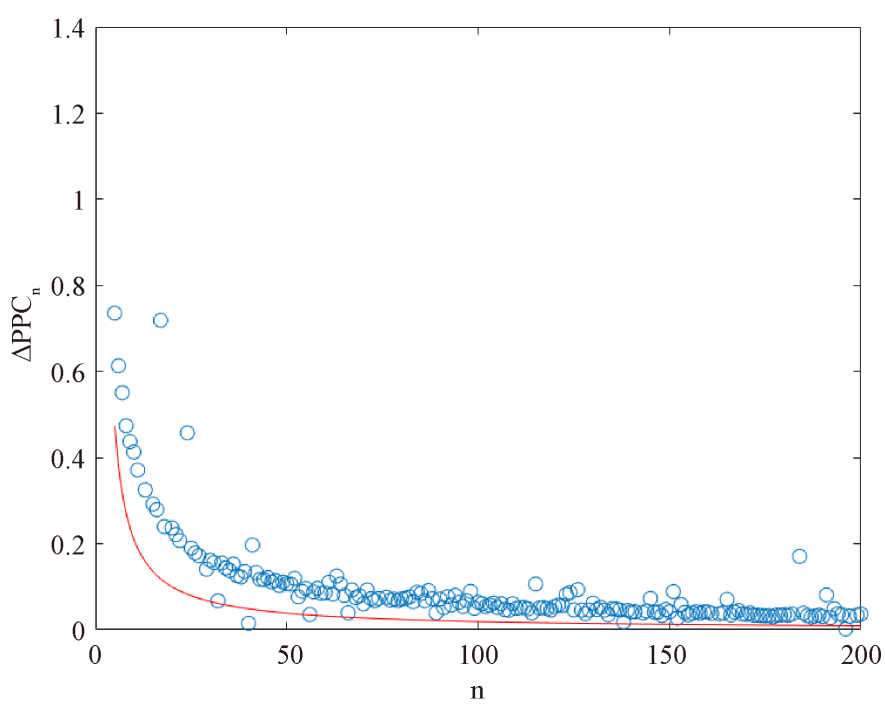

(c) $p=0.05$ 时 $\triangle P C C_{n}$ 随 $n$ 的变化情况

图 6. (a)展示的是 $n=100$ 时 $\triangle P C C_{n}$ 的分布(红线)和正态分布(蓝线)的概率密度函数, (b)展示的是 $n=1000$ 时 $\triangle P C C_{n}$ 的分布(红线)和正态分布(蓝线)的概率密度函数。(c) 展示的是当 $p$ 值取 0.05 时 $\triangle P C C_{n}$ 随着 $n$ 增加而改变的曲线, 其中横坐标代表 $n$, 纵 坐标代表在对应的 $n$ 的值以及 $p$ 值等于 0.05 的时候所对应的 $\triangle P C C_{n}$ 的值, 红色曲线 是理论上的曲线, 蓝点曲线代表随机模拟得到的曲线。

大的时候, 随机模拟曲线和理论曲线相差不大。因此 $u$ 统计量的合理性得以 验证, 于是 SSN 方法理论上的合理性也得以验证。

\section{3. 数据获取和预处理}

\section{1. 背景知识}

\subsubsection{String 网页工具}

在 String 网页工具(https://string-db.org/)中导入一列蛋白质的名称就能导出它 们之间相互作用的关系以及作用的强弱, 以图的结果呈现, 也可以导出成 tsv 
等格式放入 Cytoscape 软件里操作。

\subsubsection{Cytoscape 软件}

Cytoscape 软件是本文在用于研究不同癌症的 TP53 子网表达模式中使用的软 件。具体使用过程是在使用 String 网页工具获得 tsv 文件后, 我们将 tsv 文件 导入 Cytoscape, 然后对网络进行选取我们想要的部分、更改结点和边的样式、 给相关获得边和相关缺失边分别上色。以此做出少量癌症样本的清晰明了的 TP53 子网。此外 xlsx 等格式的文件也能导入到 Cytoscape 软件中进行作图。

\subsubsection{API 应用程序接口}

API 是 Application Programming Interface 的缩写, 翻译过来就是应用程序接 口, 它是一些预先定义好的函数或是软件系统不同组成部分衔接的约定。API 有如下优点, 第一它能完成利用程序调用网站的数据或者处理数据, 第二它 能使人更加详细地了解网站内部工作机制的一些细节。如果需要画出每个癌 症样本的 SSN, 那么就要调用 String 网页工具的 API, 因为以 BRCA (乳腺浸 润癌)为例, 我们有 1100 个癌症样本, 如果通过手动在网页工具上完成, 需 要很长时间, 并且完成的都是无意义的过程重复的工作。而调用 API, 我们 只需先将每个癌症篮选出的差异基因导出到 Excel 表格中, 做一个循环就能 使电脑自动完成这些工作。此外在绘制 8 种不同癌症的各 10 个随机癌症样本 的 TP53 子网部分, 以 BRCA 为例, 我们程序的设定是编写了了重复次数为 10 次的循环, 每一次循环调用一次 String 网页工具的 API 导出一个癌症样本 的蛋白质连接的数据, 那么一次运行就能完成一种癌症 10 个癌症样本的数据 获取。总共 80 个癌症样本的数据获取也能很快解决, 这就是 API 给我们带来 的方便、快捷性。

\section{2. 数据获取和预处理过程}

TCGA 数据可以从 GDC 官网(https://portal.gdc.cancer.gov/) 获取数据, 但是 GDC 官网下载需要配合下载工具使用并且操作起来比较麻烦, 因此本文从 Firehose (http://gdac.broadinstitute.org/runs/stddata_2016_01_28/)直接下载了 8 种癌症的 RNA 测序数据。需要注意的是 Firehose 现在的数据库已经不再更新, 所以直接从官网选择 Dashboards 的 Standard Data 选项是无法进入网站获取数 据的。由于基因测序数据种类很多, 为了简便, 我们选择的均为 Illumina 公 司的 RNASeqV2 测序数据。从数据库中下载下来的是 gz 格式的压缩包文件, 我们可以解压后用 txt 打开复制到 Excel 里创建 csv 文件, 也可以用 R 语言对.gz 格式的压缩包进行处理、提取矩阵、导出到 Excel。8 种癌症的名称以及测序 数据的总样本、癌症样本、正常样本数以及参考样本数如表 1 所示。

获得样本测序数据后, 我们接着要对数据进行第一步的处理。由于样本中 正常和癌症样本是穿插出现的, 所以第一步是对正常样本以及癌症样本分类。

TCGA 样本名称如图 7 所示, 我们只需关注第四个字段(图中 01C)中的数字, 这个数字范围是 01 29, 如果在 01 09 就是癌症样本, 在 10 29 的就是正常 样本。

TCGA 数据下载下来后, 我们发现基因测序节点名称为比如: TP53|7157, 实际我们使用的只有 TP53, 因此需要用 Matlab 对每个名称进行简单地处理, 思路是将 $\mid$ 以及后面的部分全部删除即可。 
表 1. TCGA 数据库使用的数据

\begin{tabular}{|c|c|c|c|c|c|c|}
\hline 缩写 & 中文名 & 总样本数 & 癌症 & 正常 & 参考 & 数据类型 \\
\hline BRCA & 乳腺浸润癌 & 1212 & 1100 & 112 & 20 & RNASeqV2 \\
\hline GBM & 多形成性胶质细胞瘤 & 171 & 166 & 5 & 5 & RNASeqV2 \\
\hline KIRC & 肾透明细胞癌 & 606 & 534 & 72 & 20 & RNASeqV2 \\
\hline LUAD & 肺腺癌 & 576 & 517 & 59 & 20 & RNASeqV2 \\
\hline LUSC & 肺鳞癌 & 552 & 501 & 51 & 20 & RNASeqV2 \\
\hline STAD & 胃癌 & 450 & 415 & 35 & 20 & RNASeqV2 \\
\hline THCA & 甲状腺癌 & 568 & 509 & 59 & 20 & RNASeqV2 \\
\hline UCEC & 子宫内膜癌 & 381 & 370 & 11 & 11 & RNASeqV2 \\
\hline & $\underbrace{\text { TCGA-02- }}_{\text {Project }}$ & Sample & Portion & \multicolumn{2}{|c|}{$2-01$} & \\
\hline
\end{tabular}

图 7. TCGA 数据库下载下来数据样本的命名格式, 我们需要 了解这个格式才能对样本进行分类。以图中代码为例, 第一 个字段 TCGA 代表的是项目名称; 第二个字段 02 代表组织 来源代码; 第三个字段 0001 代表参与人员；第四个字段 01 代表样本号, C 代表样本序列种样本阶数; 第五个字段 01 代表部分的次序, D 代表被分析物; 第六个字段代表板的次 序; 第七个字段代表测序中心。

笁选差异基因之后我们要生成蛋白质互作网络, 这依托的是 String 网页工 具。这个网页工具能将导入的基因名称, 通过它数据库的连接关系, 来获得 我们需要的基因之间的连接关系。因为我们通过皮尔逊相关系数构建的边很 多在蛋白质互作图中实际是不存在的, 所以我们需要借助 String 这个工具获 得真实有的边, 剔除实际上不存在的蛋白质之间连接的边。具体操作是首先 选择 Multiple Protein, 然后导入需要的蛋白质名称清单, 之后由于研究的是 人类癌症, Organism 选择的就应该是 Homo Sapiens 选项。获得图后进行 confidence 值的篎选以及隐藏没有连接的基因, 之后就能导出我们需要的表格 数据, 最后利用 Cytoscape 软件就能美化蛋白质互作图或者提取我们想要研究 的一部分。

然而, 上述利用 String 网页工具的方法对于我们后续数据比较多的研究来 说不太适合, 因为在网页上直接做图每次就只能做一张蛋白质互作图, 之后 筛选 confidence 大于 0.9 的边以及去掉没有连接的结点, 最后导出 tsv 格式, 再将 tsv 格式导入 Cytoscape 软件, 这都需要鼠标点击完成, 这是一项非常耗 费时间的工作，也就使得我们没有办法完成批量处理，没办法画出我们需要 的每个癌症样本的蛋白质互作图。因此, 通过对 String 网页工具帮助文件的 查看与学习, 我们发现可以调用网站的 API 来用 Python 程序完成上述一系列 需要鼠标点击完成的工作。如图 8 就是我们调用 String 网站 API 完成的 


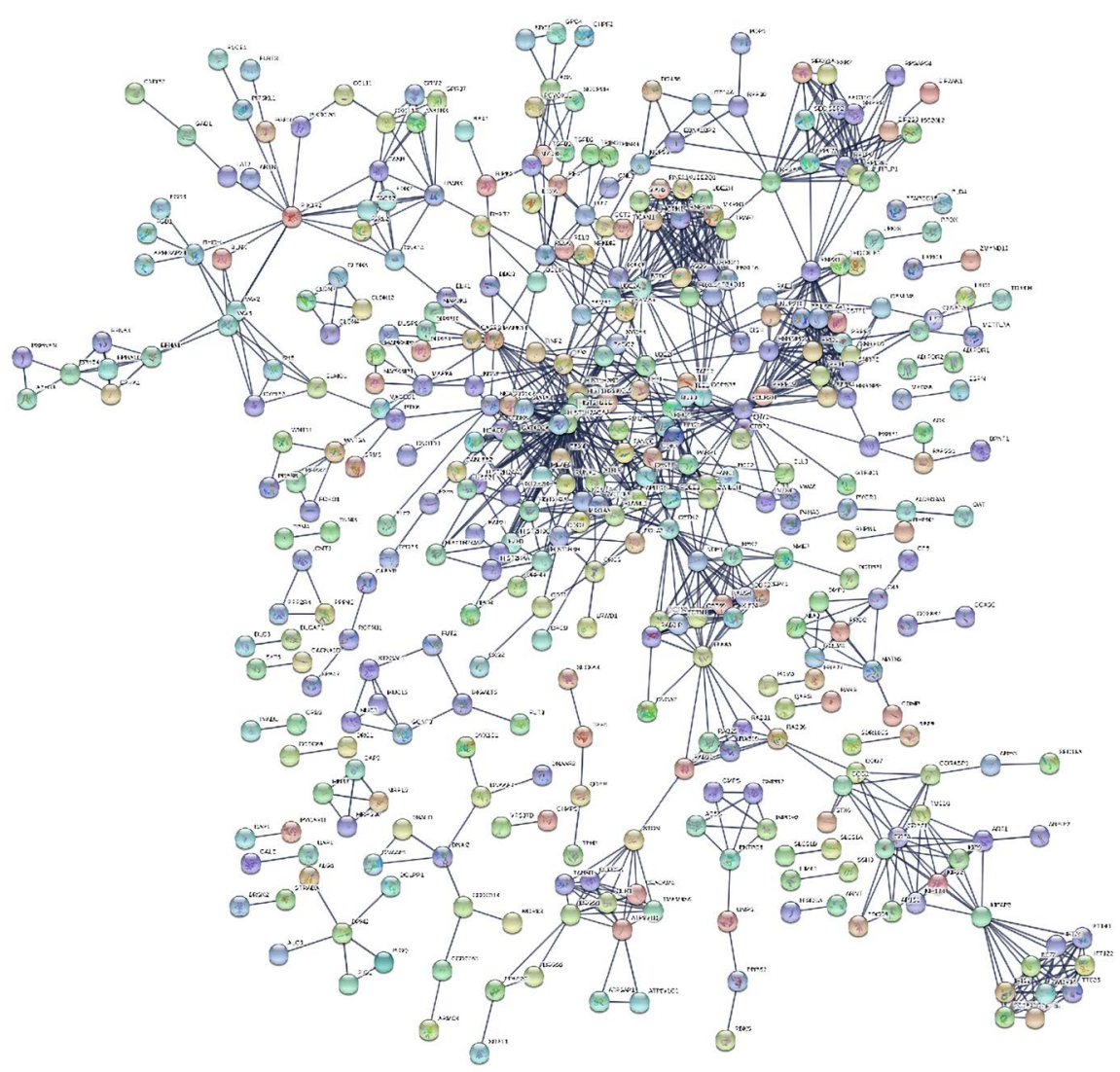

图 8. 通过筛选差异基因后, 用 Python 调用 String 网页 API 接口完成的 BRCA_A0CV 癌症样本的蛋白质互作图。其中我们只保留了 “highest confidence” 的边(结点之间连 接值大于 0.9 的边), 并且隐藏了没有边连接的结点。

BRCA_A0CV 癌症样本的蛋白质互作图。

\section{SSN可以反映出单种癌症特有的网络形式}

\section{1. 前提条件}

我们从 Firehose 下载了 8 种不同类型的癌症的数据。对于每种类型的癌症, 我们将正常样本和癌症样本进行分类, 之后取一定数量的正常样本作为参考 样本来构建参考网络。接着就能通过参考样本构建每个癌症样本的扰动网络, 以此就可以构建每个癌症样本的 SSN。为了区别癌症的网络形式, 我们首先 对 SSN 网络中的边进行一个定义、分类。这里要注意的是, 只有显著的边才 能被初步认为是 SSN 网络中的边。一条边显著的意思就是在用 $u$ 检验检验这 条边对应的 $\triangle P C C_{n}$ 与 0 是否相等的时候, 算得的 $p$ 值小于 0.05 或是一个约定 的值比如 0.01 , 即 $\triangle P C C_{n}$ 不等于 0 的边。

1、相关上调边: 即相关性增加的边, 从参考网络到扰动网络, $\triangle P C C_{n}$ 对应 的边的相关性增加, 意味着相关系数变得更接近 0 , 即 $P C C_{n}$ 的绝对值大于 $P C C_{n+1}$ 的绝对值。

$$
\left|P C C_{n+1}\right|-\left|P C C_{n}\right|<0
$$

2、相关下调边: 即相关性降低的边, 从参考网络到扰动网络, $\triangle P C C_{n}$ 对应 
的边的相关性降低, 意味着相关系数变得更远离 0 , 即 $P C C_{n}$ 的绝对值小于 $P C C_{n+1}$ 的绝对值。

$$
\left|P C C_{n+1}\right|-\left|P C C_{n}\right|>0
$$

3、相关恒定边: 相关性认为是不变的边, 那么它的 $\triangle P C C_{n}=0$, 这是不显 著的边, 不需要花时间去研究。

\subsection{TP53 子网}

为了研究不同癌症特有的网络形式, 我们选取的是 SSN 中的 TP53 子网作为 研究对象。这是由于 TP53 基因是为制造 p53 这种蛋白质提供指令的基因, 而 p53 蛋白质的作用就是抑制肿瘤。当细胞中的 DNA 受到破坏时, p53 在决定 DNA 修复还是调亡上起着关键作用。如果 DNA 可以被修复, p53 就会激活 其他基因来修复损伤。如果 DNA 不能被修复, p53 就会阻止细胞分裂并发出 信号让细胞调亡。通过阻止 DNA 突变或受损的细胞分裂, p53 有助于阻止肿 瘤的发展。TP53 的全称是 Tumor protein p53, 是著名的抑癌基因, 据资料 显示, 在超过 60 70\%, 甚至更高比例的实体瘤患者中, TP53 基因均为突变 型。此外, 在正常细胞中, 正常的 TP53 基因合成的蛋白质其实是很少的, 而 在癌细胞中, 突变后的 TP53 基因会合成大量的功能异常的 p53 蛋白质。因此, TP53 子网就是我们观察癌症疾病模式的关键部分。我们在每种癌症的癌症样 本中均通过随机的方法选取了 10 个癌症样本, 接着对 TP53 和其它结点连接 的边进行差异基因的篮选。篮选出的差异表达基因有对应的两个性质，一个 是相关上调边或是相关下调边, 相关恒定边由于不显著, 所以直接在这步就 排除掉; 另一个性质是 $p$ 值。我们用 $p$ 值从小到大排序这些笁选出的基因， 如果数量大于 1500 个, 我们就只取前 1500 个基因; 如果小于那么就取全部 个数, 这是因为 String 网页工具对检索的蛋白质数量有一定的要求, 而且数 量越多程序就越慢。10 个癌症样本篎选出的基因我们放入同一张表格的 10 个 sheet 中，例如补充文件 TP53 子网文件夹中的 BRCA_10. xlsx 文件。之后 我们调用 String 网页工具、选取 TP53 子网有的边、利用 Cytoscape 软件将相 关上调边标识为红色, 相关下调边标识为绿色, 最后以图片的形式导出。如 图 9 就是我们得到的一些 TP53 子网的图。我们总共绘制了 80 张 TP53 子网 的图, 从中观察我们可以判断出, 某些癌症的 TP53 子网几乎全是相关下调边, 比如 BRCA, 而某些癌症的 TP53 子网既有相关上调边也有相关下调边, 比如 KIRC。在 GBM 癌症中, 相关上调边和相关下调边数量基本对等。在 LUAD、

LUSC、STAD、THCA 这四种癌症中, 有的癌症样本相关上调边明显多于相 关下调边, 有的则是相关下调边明显多于相关上调边。而对于 UCEC 癌症, 在我们随机挑选的 10 个癌症样本中, 只有一个样本中有一条相关上调边, 其 余的全是相关下调边。虽然有些癌症的表达模式仅仅通过十个癌症样本无法 确切地说明出来, 但是我们任然能够发现, 不同种类癌症的 TP53 子网表达模 式是不同的, 也就是说 SSN 确实可以反应一种癌症特有的表达模式。

不同种类癌症的 TP53 子网表达模式是不同的, SSN 能反应一种癌症特有 的表达形式，这个结论仅仅通过相关上调边以及相关下调边组成的形式来得 出是不够的。因此我们还重点关注了 TP53 子网中 TP53 基因周围的临近基因。 得益于我们筛选差异基因的代码和方法, 这些临近基因具有以下两个特点: 1 、 它们都是在某种癌症中 TP53 的前 1500 位差异基因。2、它们与 TP53 基因是 


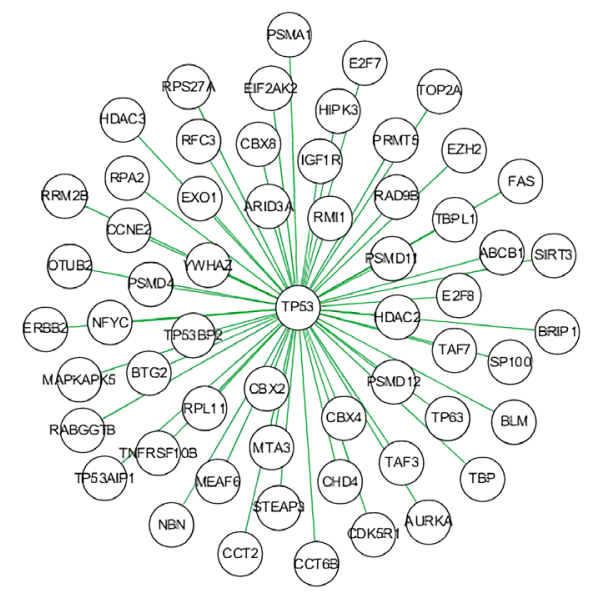

(a) BRCA 样本示例 1

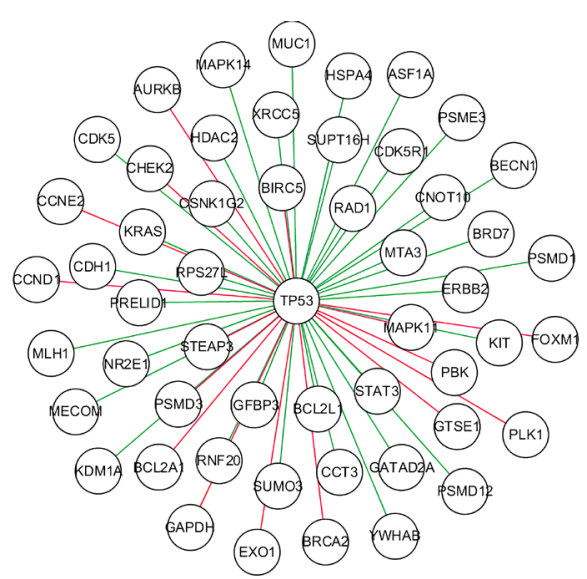

(c) KIRC 样本示例 1

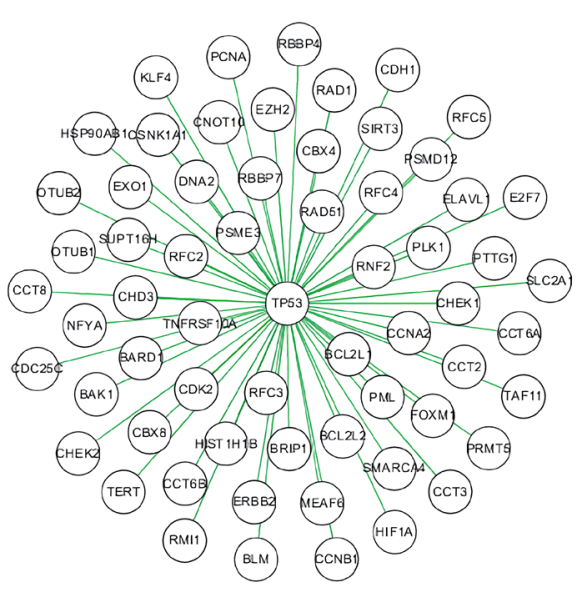

(b) BRCA 样本示例 2

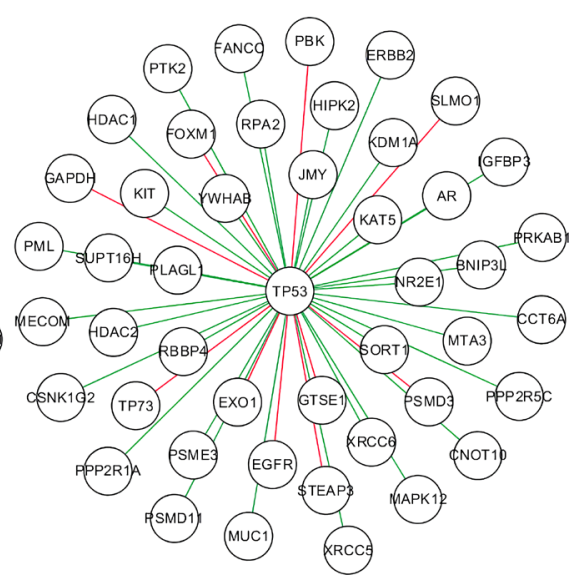

(d) KIRC 样本示例 2

图 9. 上面两张为 BRCA 中两随机癌症样本的 TP53 子网的图, 下面两张为 KIRC 中两 随机癌症样本的 TP53 子网的图。可以看到这两张图里的 BRCA 样本的 TP53 子网全 是相关下调边, 而 KIRC 样本的 TP53 子网有一些相关上调边的出现。TP53 子网共 80 张图, 均在补充文件可以看到。

真实有连接关系的, 也就是在 String 网页工具的数据库中, 它们与 TP53 基因 的 combined score (同 confidence)大于 0.85 , 这也是我们笁选的条件。由于这 些 TP53 的临近基因具有如上两个特点, 我们很容易能得出一个结论, 即这些 基因与 TP53 相关性很大。而 TP53 基因与癌症的产生有关, 因此我们不难得 到: 这些 TP53 的临近基因很可能与癌症的产生相关。接下来我们就通过分析 一些 TP53 临近基因的性质来验证我们的观点。

我们首先关注 KIRC 这种癌症。KIRC 是肾透明细胞癌的缩写。KIRC 癌症 我们也是随机挑选了 10 个癌症样本来绘制它们各自的 TP53 子网, 我们使用 样本 0 9 给这 10 个癌症样本命名。观察 KIRC 癌症中我们绘制的 10 个随机 癌症样本的 TP53 子网图, 我们发现除了样本 1 的其它 9 个样本的 TP53 子网 中, TP53 基因的临近基因都包含了 MUC1 基因。如图 10 所示为样本 6 和样 本 8 的 TP53 子网, 临近基因中的 MUC1 基因我们已用黄色标注出。查询资 料我们发现, MUC1 是一种比较有名的癌基因。同时我们可以观察到, MUC1 基因在一部分 THCA 癌症样本的 TP53 子网中也作为 TP53 的临近基因存在。 因此我们可以大胆地猜测: MUC1 基因和 KIRC 以及 THCA 癌症的产生相关。 


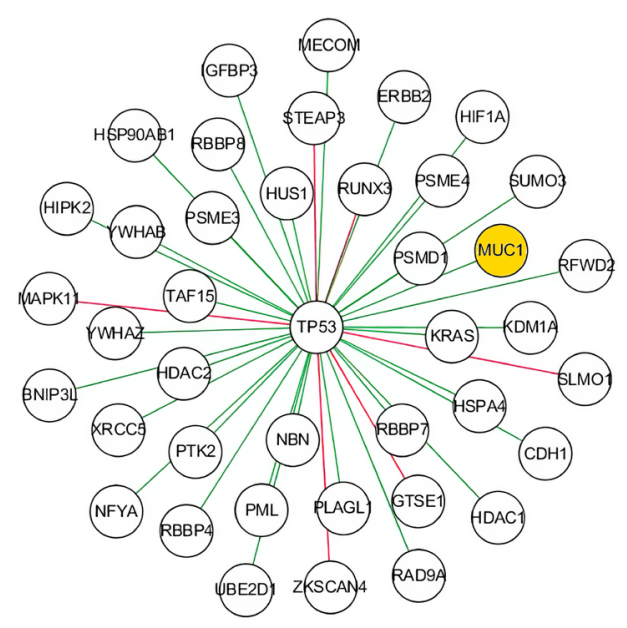

(a) KIRC 样本 6

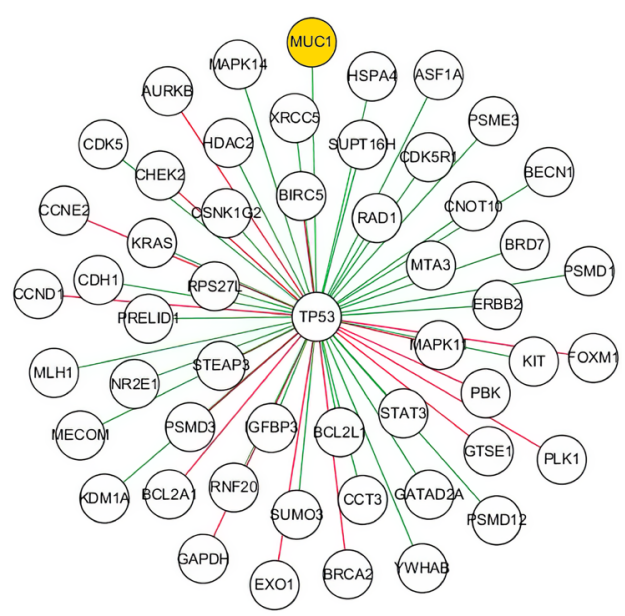

(b) KIRC 样本 8

图 10. MUC1 存在于 KIRC 癌症样本的 TP53 子 网中。

事实上有文献验证了我们的这个猜测[12] [13]。

在 GBM 癌症的 10 个随机癌症样本的 TP53 子网中, 我们发现在一些样本 的 TP53 临近基因包含了 CCNB1 基因、 RB1 基因以及 CCND1 基因, 这三种 基因均为癌基因, 与癌症的产生密不可分。

在 LUAD 癌症的 10 个随机癌症样本的 TP53 子网中, 我们发现在一些样本 的 TP53 临近基因包含了 MCL1 基因、TOP2A 基因、NGFR 基因以及 HDAC1 基因, 这四种基因均为癌基因, 与癌症的产生密不可分。

通过我们粗略的排查, 就从各个癌症的 TP53 子网中找到了与这些癌症产生 相关的基因。并且我们挑选的一般都是比较有名的癌基因, 如果一个一个基 因去挑选, 可以发现更多的癌基因。这有力地验证了我们的观点: TP53 的临 近基因很可能与癌症的产生相关。并且由于从每种癌症 TP53 基因的临近基因 挑选出来的癌基因是不全相同的, 所以我们也能得到如下结论: 在 SSN 方法 下，不同种类的癌症具有不同的、特异性的网络表达模式。

同时, 对于每个癌症样本, 我们都能发现它 TP53 子网中的有关癌基因。也 就是说 SSN 方法使得我们能做到对每个癌症病人的发病因素进行分析, 这使 
得我们对于不同个体能够针对性地靶向用药, 这也是 SSN 方法很重要的优势。

\section{3. 结论}

SSN 方法用 TP53 子网来反应 8 种癌症不同的网络表达模式。通过相关上调 边和相关下调边的分类, 首先我们确定了 TP53 子网在不同癌症下表达模式是 有差异的。接着通过分析 TP53 子网中 TP53 基因的临近基因, 我们发现这些 临近基因很可能与癌症产生相关, 并且每种癌症的 TP53 临近基因中包含的癌 基因是不太相同的。也就意味着在 SSN 方法下，不同癌症确实具有不同的网 络表达模式。对于每个癌症病人(每个样本), 我们也能通过 TP53 子网判断出 个体与癌症产生相关的突变的基因, 做到了样本特异性, 使得医疗能够做到 针对个体的个性化医疗。

\section{5. 正常样本和癌症样本的分类}

\section{1. 使用工具}

\subsubsection{NetworkAnalyst 网页工具}

我们使用 NetworkAnalyst 网页工具(https://www.networkanalyst.ca/)来实现传 统网络方法对差异基因的篮选。这个网页工具的使用非常简单明了, 只需按 照网页给出的步骤一步一步分析, 就能得到差异基因以及它们对应的 FoldChange、 $P$ 值等等性质的表格。使得我们不借助 $\mathrm{R}$ 语言编程就实现了传 统网络方法下差异基因的篮选。

\subsubsection{Heatmapper 网页工具}

我们使用 Heatmapper 网页工具(http://www.heatmapper.ca/expression/)绘制了 SSN 方法和传统网络方法在分类癌症样本时的热图以及样本树状图, 用以比 较两种方法分类的准确性。

\section{2. 传统网络方法对正常样本和癌症样本的分类}

传统网络方法是将正常样本和癌症样本全部分类放在一个矩阵。每一行代表 一个基因测序结点, 通过逐行计算正常样本和癌症样本之间值的倍数 FoldChance 简记为 FC, 一般使用的形式是它的对数形式 $\log _{2} F C$ 和检验 $p$ 值, 然后通过框定 $\log _{2} F C$ 和 $p$ 值范围篮选出关键的差异基因:

$$
\begin{gathered}
\left|\log _{2} F C\right|>1 \\
P<0.05
\end{gathered}
$$

这里我们选取了 STAD 癌症的 220 个样本, 其中包含了 35 个正常样本和 185 个癌症样本。筛选出的差异基因我们根据 $p$ 值从小到大排列, 发现 $p$ 值 等于 0 的差异基因都有很多。接着我们对 $p$ 值等于 0 的差异基因进行 $F C$ 的 从大到小排列, 以此筛选出前五位的差异基因。随后我们提取只含这五个差 异基因的表达矩阵, 放入 Heatmapper 网页工具进行热图的制作。这里需要主 义的是 Heatmapper 对表达矩阵的格式有一些要求, 我们需要在表格第一行第 一列输入 “NAME”, 最终热图格式上才不会有问题。此外, 我们利用传统 方法篮选差异基因用的是 NetworkAnalyst 网页工具。我们首先选择 NetworkAnalyst 网页工具中的 Gene Expression Table 选项, 之后导入 220 个 
样本的表达矩阵, 设置导入的 Organism 条件为 Homo Sapiens, Data type 条 件为 RNA-seq data, ID type 条件为 Official Gene Symbol, Gene-level summarization 条件为 Mean。提交数据完成后, 我们点击 proceed 进入下一步 的数据处理。简单检查下样本数量、基因数量后, 进入下一步。Filtering 选项 我们就按默认设置, 由于我们的数据从 TCGA 数据库下载下来的时候已经是 正态化数据, 因此 Normalization 选项选择 None, 提交后进入下一步。Statistical method 我们选择 Limma 包, Primary factor 选择预先定义好的 CLass。这里简 单介绍下定义的 Class, 就是在每个样本的样本名下边加一行 Normal 或者是 Tumor 的分类, 这项工作在 Excel 里直接完成。之后选择 Normal versus Tumor, 提交进入下一步后, 我们就能发现我们获得了一张差异基因的排序表格。由 于我们只需要前 5 位差异基因的热图, 而 NetworkAnalyst 网页工具之后的步 骤给出的是包含所有差异基因的热图, 所以我们将前五位基因名称记下以后, 使用 Excel 表格制作只含这五位基因的样本表达数据。至此我们获得了前五 位的差异基因和它们的样本表达数据, 接下来我们要通过这些数据完成热图 的制作。

我们借助 Heatmapper 网页工具来绘制热图。首先导入之前得到的 220 个样 本前五位差异基因的表达数据, 如果 xlsx 格式显示导入错误的话, 不妨使用 txt 格式导入。Scale Type 选择 Column, Color Brightness 选择 50, Color Scheme 选择 Custom 之后, 我们定义基因高表达的地方为红色, 低表达的地方为绿色, 中间颜色为黑色。分类方法选择 Average Linkage, 使用欧拉距离, 接着将聚 类应用到行以及列上面, 同时行列的树状图也全部显示。如此我们就得到了 传统网络方法下分类正常样本和癌症样本的热图以及样本分类情况的树状图。 我们使用这两个网页工具的原因是, 可以不用借助 $\mathrm{R}$ 语言编程来实现热图的 制作, 直接点击鼠标就能完成, 非常方便。

导出热图以及样本分类的树状图后, 我们通过数树状图中错误分类的样本, 得到 220 个样本中有 4 个样本的分类不正确, 以此计算出准确率约为 $98.2 \%$ 。 由传统网络方法得到的热图如图 11 所示。其中红色代表高表达基因, 越红代 表表达量越高; 绿色代表低表达基因, 越绿代表表达量越低。

\subsection{SSN 方法对正常样本和癌症样本的分类}

与传统网络方法对正常样本和癌症样本分类的方法不同之处就在于篮选差异 基因的方法, 其它制作热图、计算准确率的过程完全相同。为了笁选差异基 因, 这里我们使用 Matlab 编程实现, 具体代码详见补充文件或是附录。具体 思路是 35 个正常样本作为对照, 形成参考网络。之后依次构建 185 个癌症样 本的 SSN, 如果一个癌症样本里某个基因節选出来 $p$ 值小于 0.05 , 也就是意 味着这是差异基因, 那么给这个基因的计数加 1 。最后统计出在 185 个 SSN 里, 所有基因是差异基因的次数。次数从高到低排序, 以此就能获得前五位 的差异基因, 表 2 是前五位差异基因出现的次数统计。之后同传统网络方法 分类的做法一样, 我们将 220 个样本的前五位差异基因的表达数据导入到 Heatmapper 网页工具, 以此做出热图以及样本分类的树状图。根据统计 220 个样本里有 3 个样本没有被正确分类, 那么得到 SSN 方法分类正常样本和癌 症样本的准确率约为 98.6\%。由 SSN 方法得到的热图如图 12 所示。其中红色 代表高表达基因, 越红代表表达量越高; 绿色代表低表达基因, 越绿代表表 达量越低。 


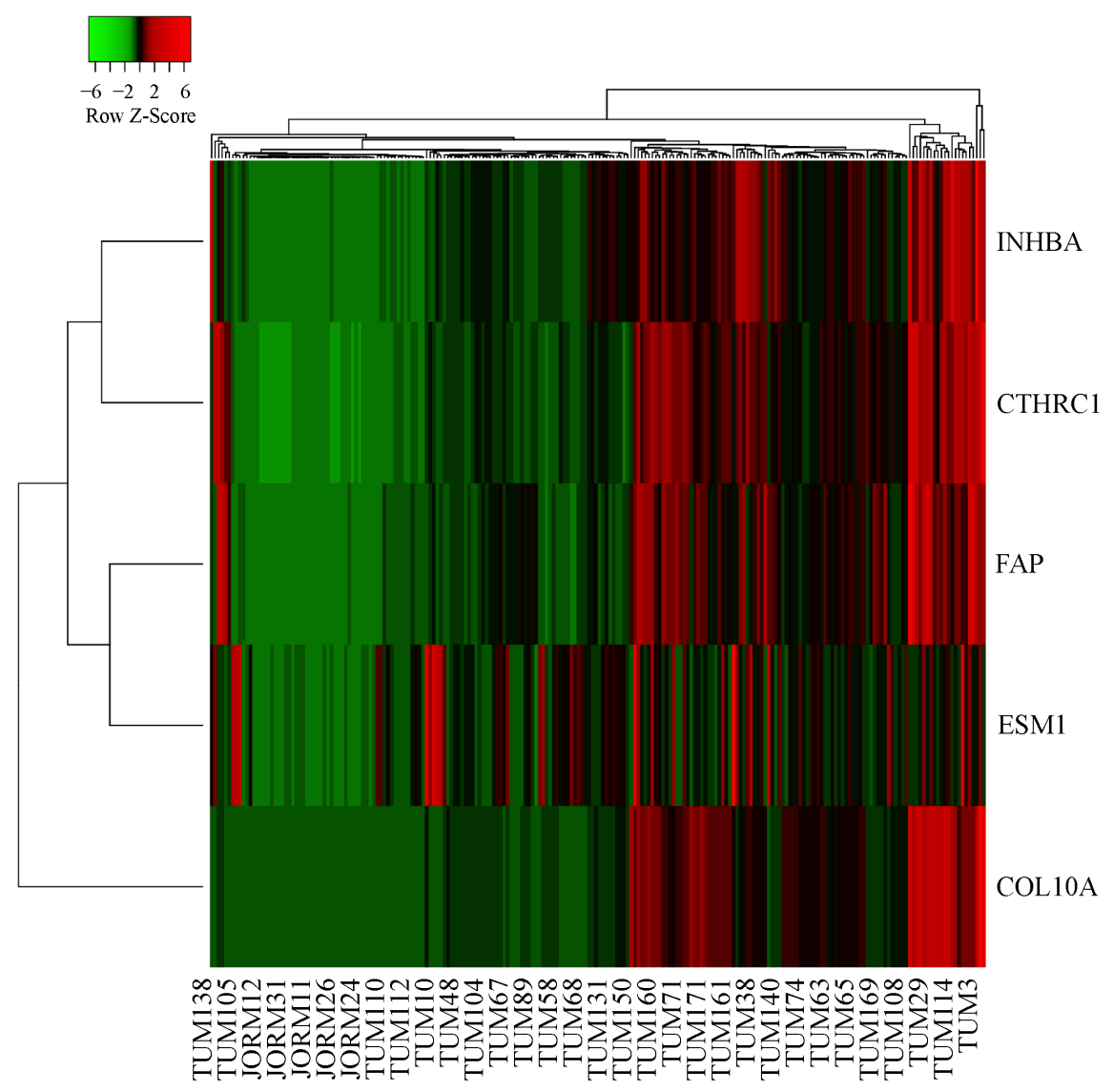

图 11. 传统网络方法分类热图。

表 2.SSN 方法節选前五差异基因。

\begin{tabular}{cc}
\hline 基因名 & 在 185 个 SSN 中显著的次数 \\
\hline COL10A1 & 179 次 \\
COL11A1 & 174 次 \\
CTHRC1 & 171 次 \\
ESM1 & 171 次 \\
INHBA & 171 次 \\
\hline
\end{tabular}

\section{4. 结论}

基于 STAD 癌症, 我们完成了传统网络方法以及 SSN 方法对正常样本和癌症 样本的分类。从实验结果上来看, 基于 SSN 方法的分类能够提高样本分类的 准确率。

比较传统网络方法和 SSN 方法, 我们可以看出在篎选前五位差异基因的时 候,两个方法仅仅具有一种基因的不同。传统网络方法篎选出的是 FAP 基因, 而 SSN 方法篮选出的是 COL11A1 基因。在热图方面, 传统网络方法将低表 达的部分聚类在了几乎最左边的位置, SSN 方法将低表达部分聚类在了靠左 的位置。而这些低表达的区域正是正常样本的基因所对应的位置, 以此我们 能看出个体如果患有 STAD 癌症, 那么这 5 种差异基因很可能不是低表达的。 


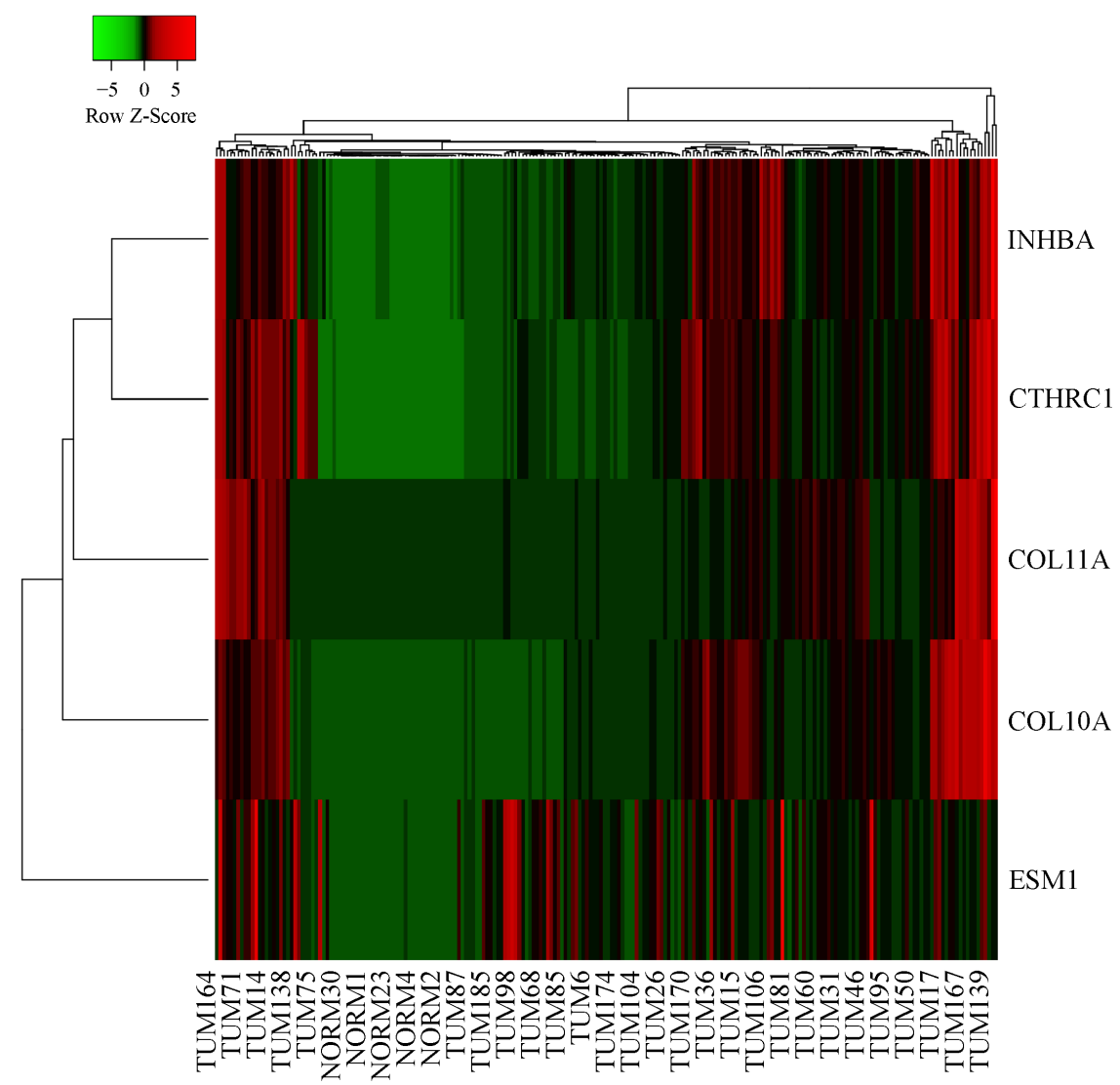

图 12. SSN 方法分类热图。

换句话说如果某个样本的这五种基因高表达了, 那么这个样本很可能就是癌 症样本。我们将正常样本按照 NOM1-35 的顺序命名, 将癌症样本按照 TUM1-185 的顺序命名。那么在数树状图计算分类准确率的时候, 我们得到 SSN 方法分类时, TUM20、TUM156、TUM101 基因分类错误。而传统网络 方法分类时, NORM25、TUM156、TUM101、TUM88 基因分类错误。由此 我们得到基于 STAD 癌症, 使用 SSN 方法能够提高样本分类的准确率, 并且 准确率较高。

\section{6. 结论与展望}

完成了以上所有工作后, 我们认为 SSN 方法在反应癌症表达模式、寻找癌症 产生的关键基因以及分类正常样本和癌症样本上是可行的, 并且可靠的。此 外, SSN 方法还具有传统网络方法不具备的优势, 即 SSN 方法可以对每个癌 症样本的关键癌症相关基因进行笁选, 并且判断每个癌症样本的癌症疾病模 式，以此实现医疗上能够针对不同个体进行个体化的医疗。

在篎选某一种癌症所有的差异基因时, 传统网络方法总共只经过了一次总 的 FoldChange 和 $p$ 值或是修正 $p$ 值的篎选, 而 SSN 方法有几个癌症样本就 需要䇥选几次, 再经过统计每个基因是差异基因的次数, 这样做无疑比传统 网络方法更准确。但是实际上目前的传统网络方法篮选差异基因是经过改进 的, 我们了解到 $\mathrm{R}$ 语言里有三个包能够完成差异基因的節选, 第一个是基于 线性模型建模的 Limma 包[14], 第二个是使用经验贝叶斯估计和基于负二项 
模型的精确检验的 EdgeR 包[15], 第三个是使用类似于 EdgeR 的负二项模型 的 DESeq 包[16]。实际使用这些包时, 也能准确篎选出差异基因。此外, 由 于对于每个癌症样本都要构建它的 SSN, 这相比于传统网络方法增加了很大 的工作量, 使得 SSN 方法程序的运行时间会增加很多。因此, 我们得出如下 结论: 师选差异基因的方法有很多, 每个方法都有它的优势和弊端, 只要合 理使用都能发挥出这些方法的价值。

对于构建生物网络, 我们认为未来会出现更多更好的办法, 会不仅比现有 的网络方法更准确, 还能一定程度上降低工作量。研究 SSN 的意义不仅在于 对传统方法的改进和提升以及个体医疗化的实现, 还在于提出一个构建生物 网络的新思路。而研究构建生物网络的意义, 就在于推动对于癌症研究的发 展, 找出更多关键的癌基因, 为人类日后研究透彻、甚至治愈癌症提供道路。

\section{致 谢}

本课题在选题及研究过程中得到程晓青老师的亲切关怀和悉心指导。她严肃 的科学态度、严谨的治学精神以及精益求精的工作作风深深地感染和激励着 我。从课题的选择到项目的最终完成, 程老师都始终给予我细心的指导和不 懈的支持，在此谨向程老师致以诚挚的谢意和崇高的敬意。

\section{References}

[1] 戴玉锦. 癌症发生机理的研究进展 [J]. 生物学杂志, 2004, 21(6): 4-7.

[2] 乔治约翰逊. 癌症机制: 越研究越复杂[J]. 环球科学, 2013(12): 88-91.

[3] Liu, X., Wang, Y., Ji, H., et al. (2016) Personalized Characterization of Diseases Using Sample-Specific Networks. Nucleic Acids Research, 44, e164. https://doi.org/10.1093/nar/gkw772

[4] Schuster, S.C. (2008) Next-Generation Sequencing Transforms Today's Biology. Nature Methods, 5, 16-18. https://doi.org/10.1038/nmeth1156

[5] 谢龙祥, 间中义, 党艺方, 等. TCGA 数据库: 海量癌症数据的源泉 [J]. 河南大学 学报(医学版), 2018(3): 223-228.

[6] Szklarczyk, D., Gable, A.L., Lyon, D., et al. (2019) STRING v11: Protein-Protein Association Networks with Increased Coverage, Supporting Functional Discovery in Genome-Wide Experimental Datasets. Nucleic Acids Research, 47, D607-D613. https://doi.org/10.1093/nar/gky1131

[7] Shannon, P., Markiel, A., Ozier, O., et al. (2003) Cytoscape: A Software Environment for Integrated Models of Biomolecular Interaction Networks. Genome Research, 13, 2498-2504. https://doi.org/10.1101/gr.1239303

[8] Guimaraes, D.P. and Hainaut, P. (2002) TP53: A Key Gene in Human Cancer. Biochimie, 84, 83-93. https://doi.org/10.1016/S0300-9084(01)01356-6

[9] Parikh, N., Hilsenbeck, S., Creighton, C.J., et al. (2014) Effects of TP53 Mutational Status on Gene Expression Patterns across 10 Human Cancer Types. The Journal of Pathology, 232, 522-533. https://doi.org/10.1002/path.4321

[10] Xia, J., Lyle, N.H., Mayer, M.L., et al. (2013) INVEX-A Web-Based Tool for Integrative Visualization of Expression Data. Bioinformatics, 29, 3232-3234. https://doi.org/10.1093/bioinformatics/btt562

[11] Babicki, S., Arndt, D., Marcu, A., et al. (2016) Heatmapper: Web-Enabled Heat Mapping for All. Nucleic Acids Research, 44, W147-W153. 
https://doi.org/10.1093/nar/gkw419

[12] 李威武, 李培军, 张宁妹, 等. MUC1 在肾癌中的表达及意义[J]. 宁夏医学杂志, 2009, 31(6): 511-512.

[13] 袁时芳, 王岭, 李开宗, 等. MUC1 在甲状腺癌及甲状腺良性病变组织中的表达 及意义[J]. 中华普通外科杂志, 2003, 8(18): 488-490.

[14] Ritchie, M.E., Phipson, B., Wu, D.I., et al. (2015) Limma Powers Differential Expression Analyses for RNA-Sequencing and Microarray Studies. Nucleic Acids Research, 43, e47. https://doi.org/10.1093/nar/gkv007

[15] Robinson, M.D., McCarthy, D.J. and Smyth, G.K. (2010) edgeR: A Bioconductor Package for Differential Expression Analysis of Digital Gene Expression Data. Bioinformatics, 26, 139-140. https://doi.org/10.1093/bioinformatics/btp616

[16] Anders, S. and Huber, W. (2012) Differential Expression of RNA-Seq Data at the Gene Level-The DESeq Package. European Molecular Biology Laboratory (EMBL), Heidelberg, 10: f1000research. 